\title{
Performance Evaluation of Various Segmentation Techniques on MRI of Brain Tissue
}

\author{
U. V. Suryawanshi \\ Dept. of Computer Science \\ College of CS \& IT, Latur, \\ Maharashtra
}

\author{
S. S. Chowhan, PhD \\ School of Computer Science \\ S. R. T. M. University, Nanded, \\ Maharashtra
}

\author{
U. V. Kulkarni, PhD \\ Dept. of CS \& Engineering \\ SGGSIE\&T, Nanded \\ Maharashtra
}

\begin{abstract}
Accuracy of segmentation methods is of great importance in brain image analysis. Tissue classification in Magnetic Resonance brain images (MRI) is an important issue in the analysis of several brain dementias. This paper reviews the performance of segmentation techniques that are used on Brain MRI. A large variety of algorithms for segmentation of Brain MRI have been developed. This paper aims at to study the performance segmentation process on MR images of the human brain, using Fuzzy c-means (FCM), Kernel based Fuzzy c-means clustering (KFCM), Spatial Fuzzy c-means (SFCM) and Improved Fuzzy c-means (IFCM). The review covers imaging modalities, MRI and methods for noise reduction and segmentation approaches. After applying all methods on MRI brain images, which are degraded by saltpepper noise, it is demonstrated that the IFCM algorithm performs more robust to noise than the standard FCM algorithm. We conclude with the trend of future research in brain segmentation by changing norms in FCM, for better results.
\end{abstract}

\section{Keywords}

Image Segmentation, Preprocessing, MRI, FCM, KFCM, SFCM and IFCM

\section{INTRODUCTION}

The study of several brain disorders, like multiple sclerosis, tumors, epilepsy, Parkinson's disease or schizophrenia or autism, involves accurate tissue segmentation from MR images of the brain. Manual tracing of white matter (WM), gray matter (GM), and cerebro-spinal fluid (CSF) in MR images by a Neurologist takes a long time for studies involving large amounts of data and is likely to show large intra- and interobserver inconsistency.

Image segmentation is a wide and dynamic field, not only in medical imaging, but also in computer vision and satellite imagery. Segmentation is a vital step to the description, recognition or classification of an image. Brain image segmentation from MR images is complicated and challenging. Its specific and exact segmentation is necessary for detection of tumors with their classification. It also helps in detection of edema, hemorrhage and necrotic tissues. For on time detection of abnormalities in brain parts, MRI is the most competent imaging technique. Unlike computerized Tomography (CT), MR images acquisition parameters can be adjusted for generating high contrast image with different gray scale level for various cases of neuropathologies [1]. Therefore, it makes role of MRI segmentation in the upcoming research limelight in medical imaging field helpful. In the field of neuroscience, mapping of functional activation of brain anatomy, the study of brain development and the analysis of neuroanatomical changeability in normal brain requires the correct identification of brain structures in
MR images [2]. Classification can be performed manually on MR images by simply selecting suitable image intensity ranges which encompass most of the voxel intensities of a particular

tissue type. On the other hand, this manual selection of thresholds is very subjective. The intensity value of the same kind of tissue may possibly dissimilar at a large range. It is the primary difficulty to get an accurate segmentation. Brain image segmentation methods are broadly classified into following categories based on [3], Thresholding approaches, Region growing approaches, classifiers, clustering approaches, Markov random field models, artificial neural networks, deformable models, and atlas guided approaches.

MRI brain image segmentation gets considerably benefit from fuzzy clustering methods from above methods. The data of MR image widely presented so far appears to be quite uncertain [4-7]. Clustering is the principally accepted method for medical image segmentation.

\section{RELATED WORK}

Vast amount of work related to various forms of image segmentation techniques including FCM are found in the literature. Let us summarize some of them.

Li [8] classified a knowledge-based classification and tissue labeling approach in FCM algorithm to mainly segment MR brain images, and introduced an expert system to find a landmark tissue by matching it with a prior model.

FCM appears to be better on normal brains, but worse on abnormal brains, according to Hall [9]. Pham and prince [3] extended the traditional FCM algorithm to deal with degraded MR images by homogeneities. FCM proves to be quiet inadequate owing to its over-sensitivity to noise, which is also, is a flaw of many other intensity-based segmentation methods. Significant uncertainty and unknown noise in the medical images generally leads to further degradation with segmentation. To overcome the noise sensitiveness of conventional FCM, Yong Yang uses [20] a novel extended FCM algorithm for image segmentation. The algorithm is developed by modifying the objective function of the standard FCM algorithm with a penalty term that takes into account the influence of the neighboring pixels on the centre pixels. Pham [25] modified the objective function in the FCM algorithm to include a multiplier field. Ahmed [4] modified the objective function of the standard FCM algorithm to allow the labels in the immediate neighborhood of a pixel to influence its labeling. The modified FCM algorithm does have an upper hand when it comes to the results of old conventional FCM methods on noisy images. However, their application to single-feature inputs faces limitation due to the way in which they incorporate the neighboring information. A FCM algorithm that incorporates spatial information into the membership function for 
clustering has been presented by Keh-Shih Chuang [21]. The spatial function is the summation of the membership function in the neighborhood of each pixel of image. Non-supervised techniques [11] such as K-means algorithm [12], minimum distance, maximum and hierarchical clustering [13] can also be found. Possibly K-means algorithm is the most widely used non-supervised technique. Many attempts have been made to develop the performance of the basic, considering pros \& cons of each technique. Aimin Yang [22] introduced KFCM-based fuzzy classifier, accuracy of which is comparable to the maximum accuracy of the multilayer neural network classifier and the relational reference with shorting down the training time. Multi-spectral brain MRI segmentation algorithm based on kernel clustering analysis has been proposed by Xiao-li Jin [23]. The algorithm, nemed multi-spectral kernel based fuzzy c-means clustering (MS-KFCM) has better segmentation performance than traditional single-channel FCM and KFCM algorithms. For betterment of results, inclusion of spatial information into the original FCM algorithm has recently been done by many researchers [25-28]. A fuzzy rule-based system to impose spatial continuity on FCM has been projected by Tolias and Panas [26]. They also used a small positive constant to modify the membership of the center pixel in a $3 \times 3$ window, in another research article [27]. Recently, Shan Shen [6], developed a new extension of FCM algorithm, called IFCM by including two prominent parameters in segmentation, which addresses the neighborhood attraction. The first parameter is the feature attraction between neighboring pixels in the image and the second one is the relative location of the neighboring pixels. Consequently, segmentation is decided not only by the pixel's intensity but also by neighboring pixel's intensities and their location. Problem of determining optimum parameters constitutes an important part of implementing the IFCM algorithm for real applications. The attraction parameters need to be properly selected in order to prevent significant degradation of the implementation performance of IFCM, so that it achieves superior partition performance compared to the FCM. For computation of these two parameters, an artificial neural network (ANN) is used.

\section{PREPROCESSING}

Preprocessing is an essential step in enabling accurate measurement of brain structures. Due to large amount of noise and non brain region the accuracy cannot be correctly obtained in segmentation process, therefore the preprocessing techniques are used. The image intensities are firstly standardized using the pixel histograms and morphological operations like dilation and erosion are used in combination to produce a desired image processing effect. Dilation and erosion are applied to purge the non-brain regions or tissue, skull, dura from brain. The experimental result shows accuracy structure of Brain MR Images. In this paper preprocessing process is done on MRI brain images. Because of noise and variances in intensity of MRI we need to remove noise and standardize signal intensity of MR images. In our paper the standardized mean 0.04 and standard deviation 0.08 of the signal intensities are selected because they can supply the best contrasts among different tissues in MR images. After standardize signal intensity of MR images next step is non-brain region removal. The brain MRI includes the whole head image, but only the brain is required for advance work like segmentation. The non-brain region should be removed before segmentation; otherwise the final results will be affected.
The simulated brain images downloaded from Brainweb dataset [19]. (http://www.bic.mni.mcgill.ca/brainweb).

A simulated T1, T2, PD; weighted MR (181X 217X 181) image was downloaded from Brainweb dataset.

Fig. 1. (a) Shows original 100th slice of T1-weighted brain MRI. Fig. 1. (b) Shows image after skull removing using the process of "Erosion" and "Dilation" and enhanced using histogram equalization. Fig. 2. (a) Shows original 80th slice of PD weighted brain MRI. Fig. 2. (b) Shows image after skull removing. Fig. 3. (a) Shows original 80th slice of T2weighted brain MRI. Fig. 3. (a) Shows image after skull removing.

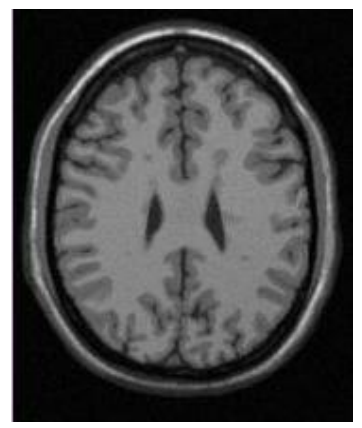

Fig. 1. (a) T1 weighted Brain MRI

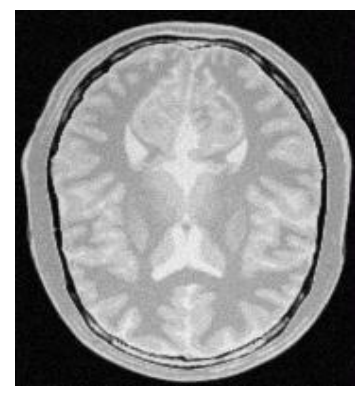

Fig. 2. (a) PD weighted Brain MRI

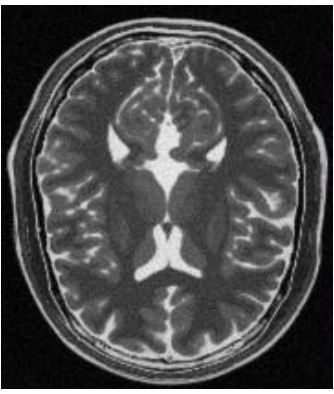

Fig. 3. (a) T2 weighted Brain MRI

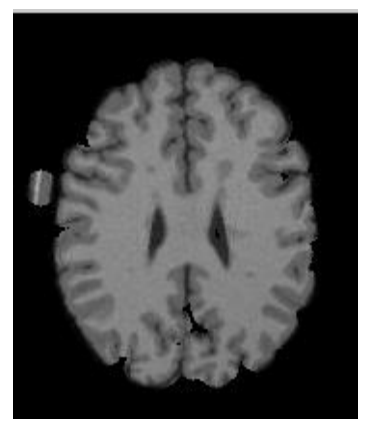

Fig. 1.(b) After removing skull

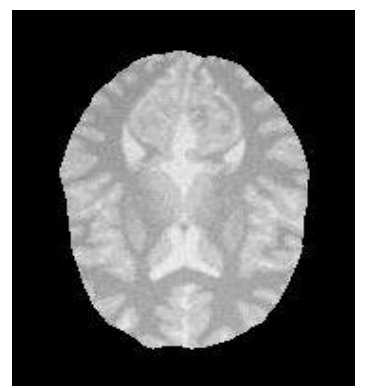

Fig. 2. (b) After removing skull

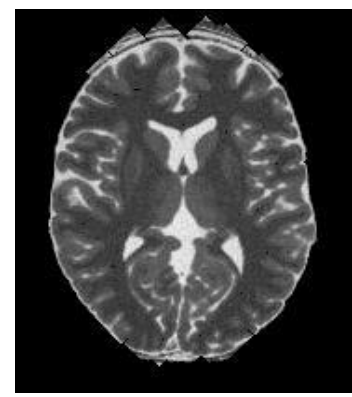

Fig. 3. (b) After removing skull

\section{MRI}

MR imaging is very useful in biological research, medical field and diagnostics. It shows excellent soft tissue contrast, non-invasive character, high spatial resolution and easy slice selection at any orientation. It uses magnetic fields and a computer to obtain an image of the brain. Electromagnetic energy produces detailed computer images of the brain from 
different angles. The MRI machine makes pictures by organizing and collecting the magnetic fields that naturally occur within the body. MRI pictures give you an idea about the soft tissues of the body (muscles, nerves, brain, discs, ligaments etc). MRI or Nuclear magnetic resonance imaging (NMRI helps to visualize the detailed structure and to study function of the body effectively. It provides detailed images of the body in any plane. MRI provides greater contrast between the different soft tissues of the body than CT does, making it especially useful in neurological (brain), musculoskeletal, cardiovascular, and cancer imaging. It is more useful than CT scan in cases which have tumors located near bony structures. It can identify brain cancer, edema (swelling of brain tissue) and hemorrhage (bleeding). A head frame, to which a MRI scanner is attached, is attached to the patient's skull, and with the aid of the computer imaging, the radiologist is able to pinpoint the tumor. MRI obtains images of your body without use X-rays or radiation, thus preventing unnecessary exposure to $\mathrm{X}$ rays. In many complications MRI gives unique information to help your doctor for deciding better plan your treatment and care. Now a day's MRI has become a most important diagnostic imaging modality, largely due to its ability to derive contrast from a number of physical parameters. It is an imaging technique used primarily in medical setting to produce high quality images of the inside of the human body. It has become very effective \& useful medical imaging technology which allows cross sectional view of the body with unprecedented tissue contrast. It has rapidly evolved into an accepted modality for medical imaging of disease processes in the musculoskeletal system, especially brain due to the use of non-ionizing radiation.

It has the added advantage of being able to produce images which slice through the brain in both horizontal and vertical planes [5-8]. It also helps in the early detection of abnormal changes in tissues and organs [2]. It possesses good contrast resolution for different tissues. Medical image contains considerable uncertainty and noise, and can lead to difficulties with segmentation. A major problem with MRI is that the signal intensities do not have a fixed value, even in the same body region of the same patient using the same scanner [4]. This indicates that the same tissue type may have different scales of signal intensity in the different images. Therefore; standardization procedure using the pixel intensity histogram is applied, to ensure the signal intensities of different tissues are uniform. Our major interest is the human brain. Non-brain regions including the skull, meninges etc, which are not required, may possibly influence the segmentation results. Morphological analysis was used to remove non-brain regions before any traditional segmentation techniques.

\section{SEGMENTATION}

Segmentation of medical images is a complicated task because many factors like noise, sampling artifacts, skull, etc affects on end result of segmentation. Image segmentation is called as exhaustive partitioning of an experimental input image into a series of regions each of which is considered to be homogeneous with respect to some image property of interest (intensity), [1]. These regions represent different human tissue structures and decide the performance of some advanced medical image processing and the accuracy of clinical diagnosis. These advanced medical image processing incorporate feature registration, anatomy structure analysis, $3 \mathrm{D}$ reconstruction, movement analysis, and etc. For segmentation, the brain tissue is divided into WM, GM and
CSF. The particular measurement of WM, GM and CSF is essential for quantitative pathological investigation. Number of methods for segmenting MRI brain image data is developed. From different methods fuzzy clustering analysis has proven to be a successful tool in image analysis [2]. The segmentation process can be split into three major steps.

[a] Pixel classification:- Pixels are classified into lot of classes. The number of classes is equivalent to the number of tissues in the brain, so that there exists a one-to-one correspondence between the classes and the tissues. Methods such as FCM, KFCM, ANN, etc. perform this task.

[b] Correction:- In this step, we remove any misclassification caused due to in homogeneities of the radio frequency and magnetic fields, imaging noise, etc. $n \times n$ majority filters and Markov Random fields are used for this purpose.

[c] Tissue labeling: - During this step, a class of pixels is assigned a unique tissue name. This is done using a prior knowledge about density-tissue relationship or by means of an anatomical atlas knowledge base. In this paper, we only concentrate on the first step, i.e. pixel classification.

\section{MRI SEGMENTATION ALGORITHMS}

Segmentation is the partitioning of an image into meaningful regions, normally to differentiate objects or regions of interest like foreground and background. There would be only these two classes foreground and background and the segmented image would be a binary image.

Some Segmentation methods are discussed here:

\subsection{Fuzzy C-Means}

The conventional FCM algorithm is a worldwide accepted image segmentation method. The segmentation of imaging data involves partitioning the image data into different cluster regions with similar intensity image values. Mostly medical images always present overlapping gray-scale intensities for different tissues. Therefore, fuzzy clustering methods are suitable for the segmentation of medical images. FCM can be seen as the fuzzified version of the $K$ - means algorithm. It is a method of clustering which allows one piece of data to belong to two or more clusters. FCM is a data clustering technique in which each sample data point belongs to a cluster to some degree that is specified by a membership grade. The FCM clustering algorithm was first introduced by DUNN [14] and later was extended by BEZDEK [15], which has been successfully applied to image segmentation by minimizing following objective function with respect to fuzzy membership, and set of cluster centroid $V$.

$$
J_{m}(U, V)=\sum_{j=1}^{N} \sum_{i=1}^{C} u_{i j}^{m} d^{2}\left(\mathbf{x}_{j}, \mathbf{v}_{i}\right)
$$

Where:

The parameter $m$ is weighting exponent on each fuzzy membership and control the degree of "fuzziness" of the resulting classification. In actual application, $m=2$ is frequently adopted. $X=\left\{\mathbf{x}_{\mathbf{1}}, \mathbf{x}_{\mathbf{2}}, \ldots \mathbf{x}_{j}, \ldots \mathbf{x}_{N}\right\}$ is a $p \times N$ data matrix, where $p$ represents the dimension of each $\mathbf{x}_{j}$ "feature" vector, and $N$ represents the number of feature 
vectors (pixel numbers in the image). $C$ is the number of clusters. $U$ is fuzzy matrix. $d\left\|\mathbf{x}_{j}-\mathbf{v}_{i}\right\|$ is the Euclidean norm. The membership function is expressed as follows:

$$
u_{i j}=\frac{1}{\sum_{k=1}^{C}\left(\frac{d\left(\mathbf{x}_{j}, \mathbf{v}_{i}\right)}{d\left(\mathbf{x}_{j}, \mathbf{v}_{i}\right)}\right)^{2 /(m-1)}}
$$

$V=\left\{\mathbf{v}_{\mathbf{1}}, \mathbf{v}_{\mathbf{2}}, \ldots \mathbf{v}_{i}, \ldots \mathbf{v}_{C}\right\}$ Which is a $p \times C$ matrix and denotes the cluster feature center

$$
\mathbf{v}_{i}=\frac{\sum_{j=1}^{N}\left(u_{i j}\right)^{m} \mathbf{x}_{j}}{\sum_{k=1}^{C}\left(\sum_{j=1}^{N} u_{i j}\right)^{m}}(i=1,2, \ldots, C)
$$

The Process stops when $\left\|J_{\mathrm{m}}{ }^{t+1}-J_{\mathrm{m}}{ }^{t}\right\|<\varepsilon$ or predefined number of iterations is reached, where as $t$ is no. of iteration.

The membership function in (2) is mostly decided by, which measures the similarity between the pixel intensity and the cluster center. Higher membership depends on closer intensity values to the cluster center. Although clustering algorithms do not require training data, they require an initial segmentation (initial parameters). Clustering algorithms do not directly incorporate spatial modeling and can therefore be sensitive to noise and intensity inhomogeneities. If an MR image contains noise, it affects on pixel intensities, which will result in an inaccurate membership and improper segmentation.

\subsection{Kernel-Based FCM}

FCM can be robust to noise and outliers when replacing a new kernel-based metric in the original Euclidean norm metric of FCM. A Kernel-Based Fuzzy C-Means clustering (KFCM) algorithm has been proposed by Zhang [16, 17] with strong noise robustness. The reason is that an exponential-type distance is bounded and monotone increasing, based on the concept of machine learning with a learning capability to improve the performance of clustering results [24].

The KFCM partitions a dataset $X=\left\{x_{1}, x_{2}, \ldots, x_{n}\right\} \subset R^{P}$, where $P$ is the dimension, into $c$ fuzzy subsets by minimizing the objective functions:

$$
J_{m}(U, V)=\sum_{i=1}^{c} \sum_{k=1}^{n} u_{i k}^{m}\left\|\Phi\left(x_{k}\right)-\Phi\left(v_{i}\right)\right\|^{2}
$$

Where $\Phi$ an implicit nonlinear map and other components are the same with equation (1). $n$ is a number of sample. $u_{i k}$ is a memberships function and $v_{i}$ is a cluster center function. In feature space, a kernel can be a function which is called $K$, where

$$
K(x, y)=\langle(\Phi(x), \Phi(y))\rangle \text { And }\langle.\rangle \text { is the inner product. }
$$

Moreover, by considering the most popular kernel, i.e.
Gaussian Radial basis function (GRBF) kernel.

$$
K(x, y)=\exp \left(-\|x-y\|^{2} / \sigma^{2} \text { Where } \sigma\right. \text { is the width }
$$

parameter, the objective function will be

$$
J_{m}(U, V)=\sum_{i=1}^{c} \sum_{k=1}^{n} u_{i k}^{m}\left[1-K\left(x_{k}-v_{i}\right)\right]
$$

KFCM should adjust some parameters like $\sigma$ as dispersion. This parameter affects KFCM results.

\subsection{Spatial FCM}

One of the imperative uniqueness of an image is that neighboring pixels have similar feature values, and the probability that they belong to the same cluster. FCM algorithm that incorporates spatial information into the membership function for clustering. The spatial function is the summation of the membership function in the neighborhood of each pixel under consideration

Chuang [7] proposed another spatial FCM algorithm in which spatial information can be incorporated into fuzzy membership function directly using

$$
\mu_{m n}=\frac{\mu_{m n}^{p} h_{m n}^{q}}{\sum_{K=1}^{C} \mu_{k n}^{p} h_{k n}^{q}}
$$

Where $p$ and $q$ are two parameters controlling the respective contribution. The variable $h_{m n}$ includes spatial information by

$$
h_{m n}=\sum_{k \in N n} u_{n k^{\prime}}
$$

Where $N n$ denotes a local window centered on the image pixel $n$, the weighted $\mu_{m n}$. The clustering is a two-pass process at each iteration. The first pass is the same as that in standard FCM to calculate the membership function in the spectral domain. In the second pass, the membership information of each pixel is mapped to the spatial domain, and the spatial function is computed from that. The FCM iteration proceeds with the new membership that is incorporated with the spatial function. The iteration is stopped when the maximum difference between two cluster centers at two successive iterations is less than a threshold $(=0.02)$. After the convergence, defuzzification is applied to assign each pixel to a specific cluster for which the membership is maximal. The spatial function modifies the membership function of a pixel according to the membership statistics of its neighborhood. Such neighboring effect biases the solution toward piecewise-homogeneous labeling. This technique reduces the number of spurious blobs, and the segmented images are more homogeneous. The SFCM algorithm with a higher $q$ parameter provides a better smoothing effect

\subsection{Improved Fuzzy C -Means}

A new algorithm is developed by Shan Shen [6] in order to avoid the drawbacks of other methods of clustering. In this paper comparison of IFCM with other methods described below. Usually, one pixel is too small to represent part of an 
image. Assuming a pixel has a completely different intensity from its surrounding pixels, it is reasonable to conclude that this pixel must be affected by noise. Its real intensity should be identical to its neighboring pixels. There is an attraction exists between neighboring pixels, which we coin "neighborhood attraction". Within IFCM algorithm, during clustering, each pixel attempts to attract its neighboring pixels toward its own cluster. If a pixel has a very similar intensity to one of its neighbors, the attraction between them is stronger than the attraction between the neighboring pixels with different intensities. A spatially closer neighboring pixel should also have a stronger attraction than a neighbor which is spatially distant. The components of the neighborhood can also influence the attraction. IFCM segmentation is not only decided by the pixel itself but also by its neighboring pixels. The membership value of FCM decides the segmentation results, and the membership value is determined by the similarity measurement $d^{2}\left(\mathbf{x}_{j}, \mathbf{v}_{i}\right)$. It may be deduced that this measurement is the key to segmentation success. In FCM, $d^{2}\left(\mathbf{x}_{j}, \mathbf{v}_{i}\right)$ is a measure of the difference between the intensity of a pixel and the cluster center, and has no resistance to noise. Neighborhood attraction is considered to exist between neighboring pixels. In clustering, each pixel attracts its neighboring pixels toward its own cluster. This neighborhood attraction depends on two factors; the pixel intensities or feature attraction, and the spatial position of the neighbors or distance attraction, which also depends on the neighborhood structure. IFCM considers the neighborhood attraction in $d^{2}\left(\mathbf{x}_{j}, \mathbf{v}_{i}\right)$ directly

$$
d^{2}\left(\mathbf{x}_{j}, \mathbf{v}_{i}\right)=\left\|\mathbf{x}_{j}-\mathbf{v}_{i}\right\|^{2}\left(1-\lambda H_{i j}-\xi F_{i j}\right)
$$

Here $H_{i j}$ represents feature attraction and $F_{i j}$ represents the distance attraction. The two parameters $\lambda$ and $\xi$ in (8) of magnitude between 0 to 1 , adjust the degree of the two neighborhood attractions

$$
H_{i j}=\frac{\sum_{k=1}^{S} u_{i k} g_{j k}}{\sum_{k=1}^{S} g_{j k}}
$$

Where $g_{j k}$ is the intensity difference between the study pixel $j$ and its neighbor pixel $k$.

$$
g_{j k}=\left|X_{j}-X_{k}\right|
$$

$$
F_{i j}=\frac{\sum_{k=1}^{s} u_{i k}^{2} q^{2} j k}{\sum_{k=1}^{s} q_{j k}^{2}}
$$

Where $q_{j k}$ is the relative location between pixel $j$ and its neighborhood pixel $k$. The neighborhood structure is of the form $K_{j}=\{k\}$

$$
K_{j}=\left\{k \in N \mid O<\left(a_{j}-a_{k}\right)^{2}+\left(b_{j}-b_{k}\right)^{2} \leq Q\right\}
$$

Whereas $\left(a_{j}-a_{k}\right),\left(b_{j}-b_{k}\right)$ denotes the coordinates of pixel $j, k, Q$ is constant equal to $2^{(L-1)}$, and $L$ is the level of the neighborhood. $q_{j k}$ in (11) can be described as follows:

$$
q_{j k}=\left(a_{j}-a_{k}\right)^{2}+\left(b_{j}-b_{k}\right)^{2}
$$

Two parameters $\lambda$ and $\xi$ are selected to adjust the degree of feature attraction and distance attraction in (6). A simple ANN model is designed to search optimal value of two parameters.

\section{RESULTS}

To conclude the paper it's been found that, IFCM is well thought-out superior than FCM, KFCM, and SFCM. To evaluate the segmentation performance, some definitions are required.

$N_{f p}$ is the number of pixels that do not belong to a cluster and are segmented into the cluster. $N_{f n}$ is the number of pixels that belong to a cluster and are not segmented into the cluster. $N_{p}$ is the number of all pixels that belong to a cluster, and $\mathrm{N}_{\mathrm{n}}$ is the total number of pixels that do not belong to a cluster. Three evaluation parameters may now be defined as follows.

1) Under segmentation $(U n S)$ :- $N_{f p} / N_{n}$ representing the percentage of negative false segmentation.

2) Over segmentation $(O v S):-N_{f n} / N_{p}$ representing the Percentage of positive false segmentation.

3) Incorrect segmentation (InC) :- $\left(N_{f p}+N_{f n}\right) / \mathrm{N}$ representing the total percentage of false segmentation.

Table I depicts the evaluation parameters of four mentioned methods when applied to the MR phantom. It clearly shows the performance rate of IFCM as compare to other methods.

Table I: Segmentation Evaluation on $T_{1}$ Weighted MR image

\begin{tabular}{|c|c|c|c|c|c|}
\hline \multirow{2}{*}{ CLASS } & $\begin{array}{l}\text { Evaluation } \\
\text { Parameters }\end{array}$ & FCM & KFCM & SFCM & IFCM \\
\hline \multirow{3}{*}{ CSF } & UnS(\%) & $\mathbf{2 . 3 9 7}$ & $\mathbf{3 . 5 9 6 6}$ & $\mathbf{3 . 5 7 5}$ & $\mathbf{3 . 0 0 0 0}$ \\
\cline { 2 - 6 } & OvS(\%) & $\mathbf{1 . 0 0 0 0}$ & $\mathbf{1 . 1 9 8 8}$ & $\mathbf{1 . 2 3 7}$ & $\mathbf{1 . 0 0 0 0 0}$ \\
\cline { 2 - 6 } & InC(\%) & $\mathbf{4 3 6 . 0 0}$ & $\mathbf{7 . 1 9 3 3}$ & $\mathbf{7 . 1 2 8}$ & $\mathbf{6 . 0 0 0 0 0}$ \\
\hline \multirow{3}{*}{$\begin{array}{l}\text { White } \\
\text { Matter }\end{array}$} & UnS(\%) & $\mathbf{3 . 5 9 6 6}$ & $\mathbf{2 . 3 9 7 7}$ & $\mathbf{2 . 4 9 6}$ & $\mathbf{2 . 0 0 0 0 0}$ \\
\cline { 2 - 6 } & OvS(\%) & $\mathbf{1 . 0 0 0 0}$ & $\mathbf{1 . 1 9 8 8}$ & $\mathbf{2 . 2 9 8}$ & $\mathbf{1 . 0 0 0 0 0}$ \\
\cline { 2 - 6 } & InC(\%) & $\mathbf{6 5 4 . 0 0}$ & $\mathbf{4 . 7 9 5 5}$ & $\mathbf{3 . 8 9 7}$ & $\mathbf{4 . 0 0 0 0 0}$ \\
\hline \multirow{2}{*}{$\begin{array}{c}\text { Gray } \\
\text { Matter }\end{array}$} & UnS(\%) & $\mathbf{1 . 1 9 8 8}$ & $\mathbf{1 . 1 9 8 8}$ & $\mathbf{1 . 1 8 9}$ & $\mathbf{1 . 0 0 0 0 0}$ \\
\cline { 2 - 6 } & OvS(\%) & $\mathbf{1 . 0 0 0 0}$ & $\mathbf{1 . 1 9 8 8}$ & $\mathbf{1 . 2 9 8}$ & $\mathbf{1 . 0 0 0 0 0}$ \\
\cline { 2 - 6 } & InC(\%) & $\mathbf{2 1 8 . 0 0}$ & $\mathbf{2 . 3 9 7 7}$ & $\mathbf{2 . 5 8 9 7}$ & $\mathbf{2 . 0 0 0 0 0}$ \\
\hline
\end{tabular}

The simulated Brain MR image consisting of white matter, cerebral spinal fluid (CSF), and gray matter. 4\% salt and pepper noise was applied to 100th slice of the T1 weighted MR image as shown in Fig. 4(a). This noisy slice was 
segmented into four clusters: background, white matter, gray matter and CSF using FCM, shown in Fig. 4(b), (c), and (d); from the result background was neglected. KFCM, SFCM, IFCM segmentation techniques are applied on noisy 100th slice of Brain MR and will get segmented clusters (white matter, gray matter and CSF) in Fig. 5(b), (c) and (d), Fig. 6(b), (c) and (d), Fig. 7(b), (c) and (d), respectively.

A simulated 80th slice of T2-weighted image was also used to verify the segmentation result of IFCM. 3\% salt and pepper noise was applied to the MR image as shown in Fig.8 (a). This noisy slice was segmented into four clusters: background, white matter, gray matter and CSF using FCM, shown in Fig. 8(b), (c), and (d); from the result background was neglected.

SFCM, IFCM segmentation techniques are applied on noisy 80th slice of T2 weighted Brain MR and will get segmented clusters (white matter, gray matter and CSF) in Fig. 9(b), (c) and (d), Fig. 10(b), (c) and (d), correspondingly.

FCM and SFCM are unable to segment the slice correctly, and noise still remains present, IFCM produced the better result with insignificant noise.

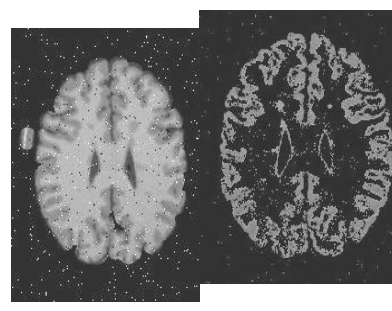

(a)

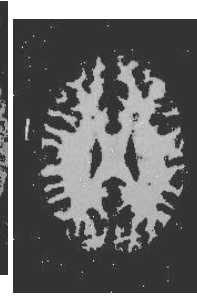

(c)

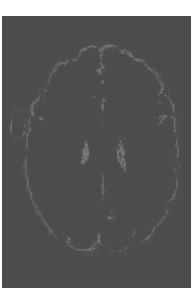

(d)
Fig. 4. Simulated 100th noisy T1 -weighted MR image. (1) Segmentation result of FCM. (b) White Matter (c) Gray Matter (d) CSF

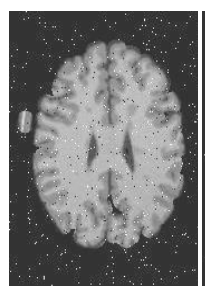

(a)

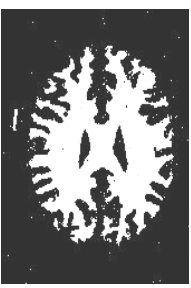

(b)

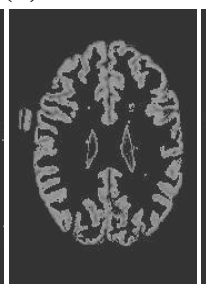

(c)

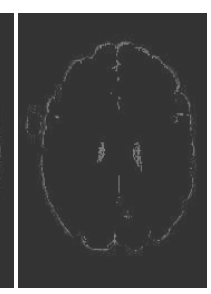

(d)
Fig. 5. Simulated 100th noisy $T 1$-weighted MR image. (2) Segmentation result of KFCM. (b) White Matter (c) Gray Matter (d) CSF

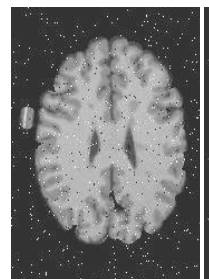

(a)

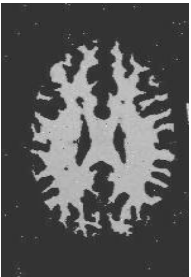

(b)

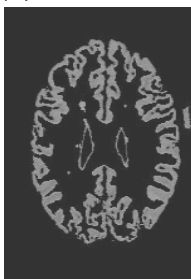

(c)

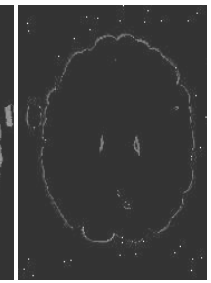

(d)
Fig. 6. Simulated 100th noisy T1 -weighted MR image. (3) Segmentation result of SFCM. (b) White Matter (c) Gray Matter (d) CSF

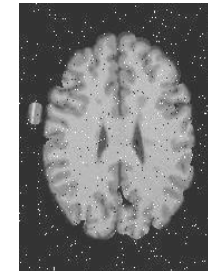

(a)

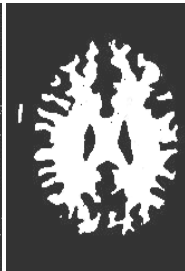

(b)

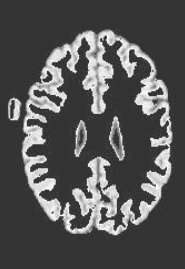

(c)

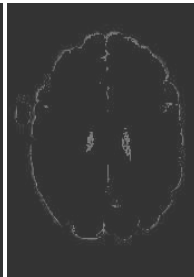

(d)
Fig. 7. Simulated 100th noisy T1 -weighted MR image. (3) Segmentation result of IFCM. (b) White Matter (c) Gray Matter (d) CSF

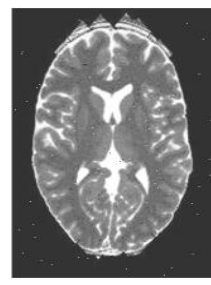

(a)

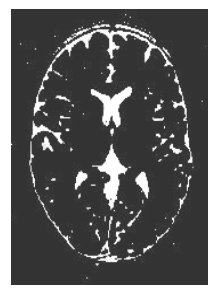

(b)

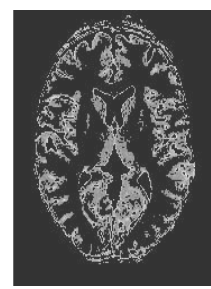

(c)

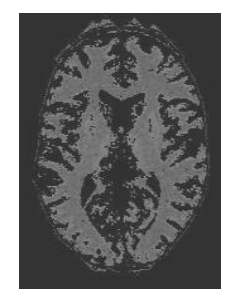

(d)
Fig. 8. Segmentation result of FCM. (a) Simulated 80th noisy T2 weighted MR image (b) CSF (c)White Matter (d) Gray Matter

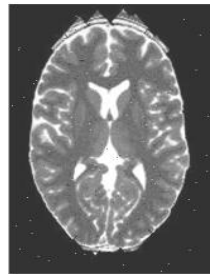

(a)

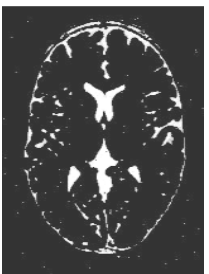

(b)

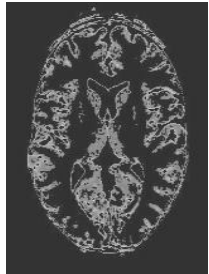

(c)

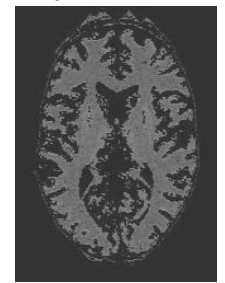

(d)
Fig. 9. Segmentation result of SFCM. (a) Simulated 80th noisy T2 weighted MR image (b) CSF (c)White Matter (d) Gray Matter

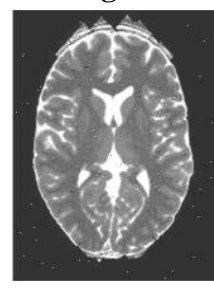

(a)

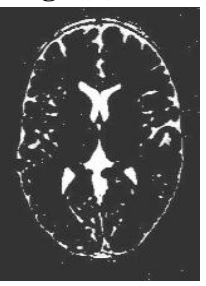

(b)

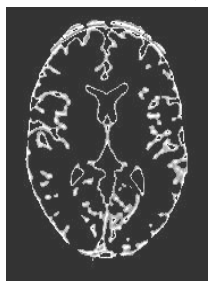

(c)

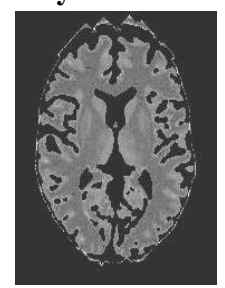

(d)
Fig. 10. Segmentation result of IFCM. (a) Simulated 80th noisy T2 weighted MR image (b) CSF (c)White Matter (d) Gray Matter

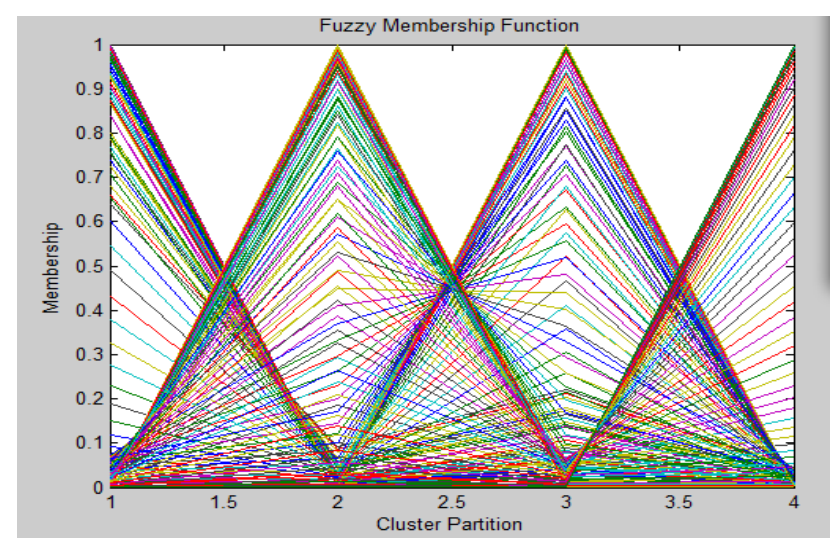

Fig. 11. Fuzzy membership functions in FCM 


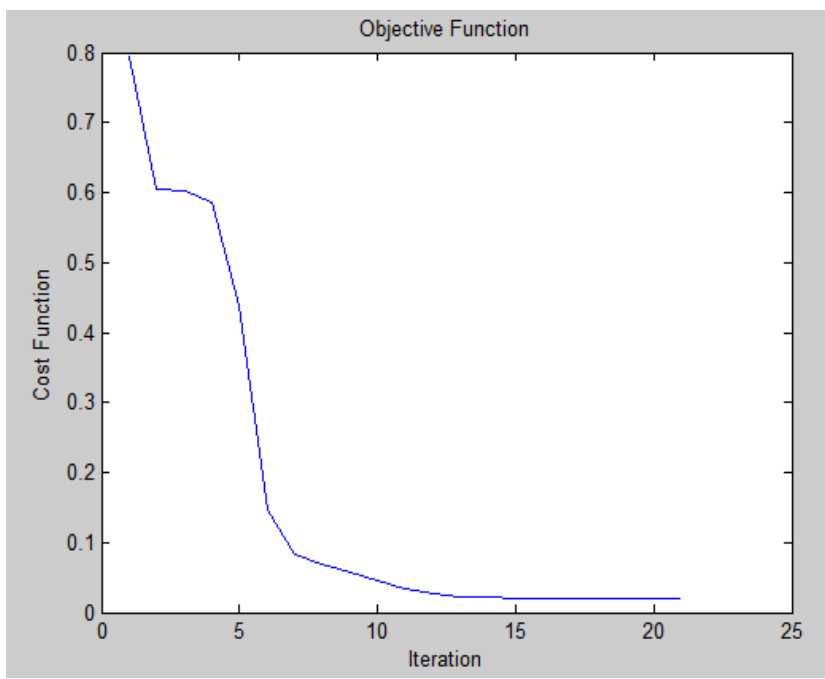

Fig. 12. Object function in FCM

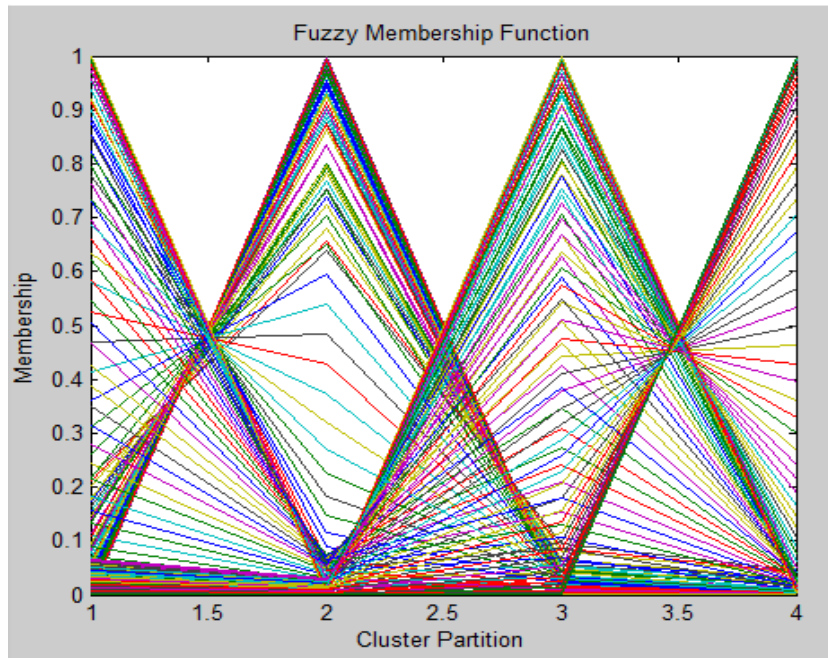

Fig. 13. Fuzzy membership function in IFCM

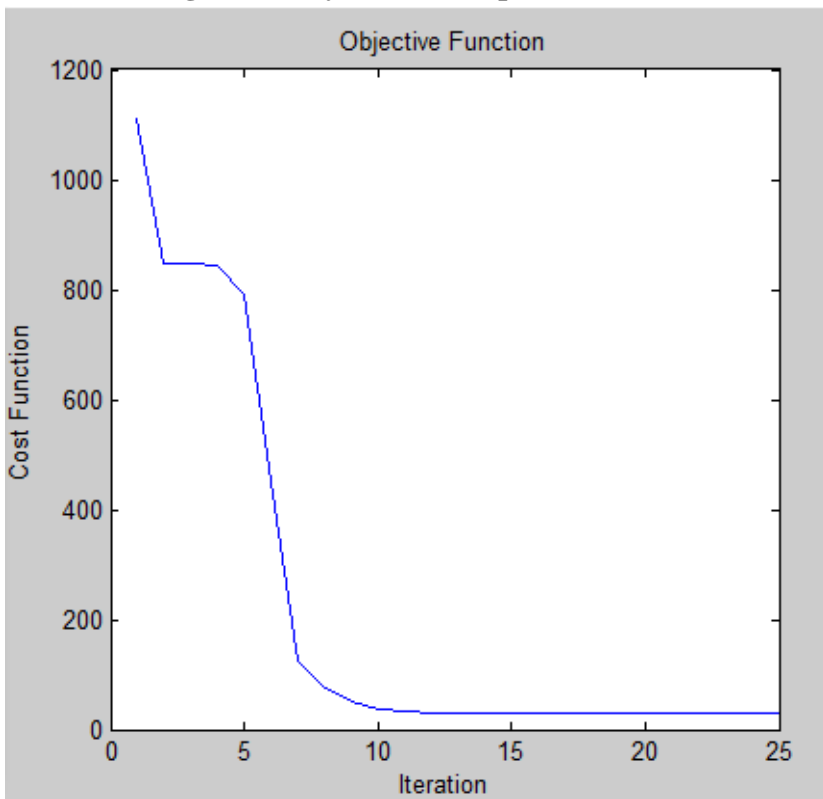

Fig. 14. Objective function in IFCM

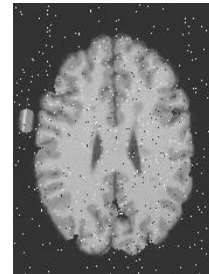

(a)

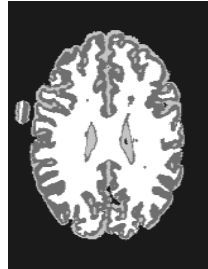

(b)

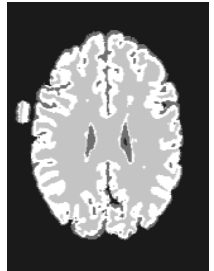

(c)

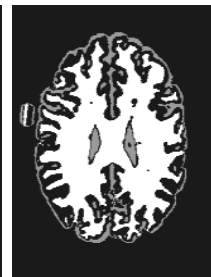

(d)

Fig.15. (a) Noisy $100{ }^{\text {th }} \mathrm{T} 1$ weighted MR image with $3 \%$ noise (b) Segmentation Result of FCM. (c)

Segmentation Result of IFCM. (d) Segmentation Result of SFCM

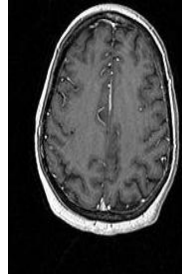

(a)

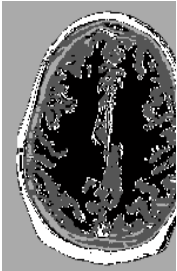

(b)

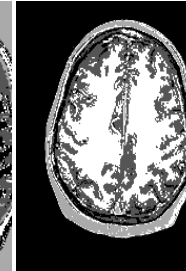

(c)

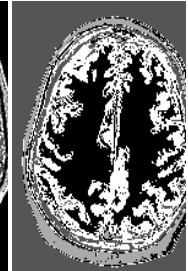

(d)

Fig. 16. (a)T1 weighted MR image. (b) Segmentation Result of FCM (c) Segmentation Result of SFCM (d) Segmentation Result of IFCM

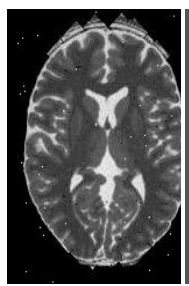

(a)

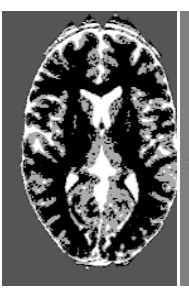

(b)

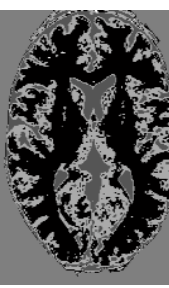

(c)

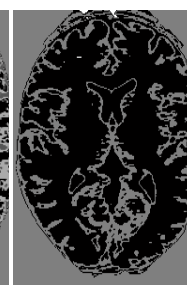

(d)

Fig. 17. (a) Noisy $80^{\text {th }} \mathrm{T} 2$ weighted MR image with 3\% noise. (b) Segmentation results of FCM. (c) Segmentation Result of SFCM. (d) Segmentation Result of IFCM

Fig. 11 and 13 shows Fuzzy membership functions in FCM and IFCM correspondingly. Fig. 12 and 14 shows objective functions in FCM and IFCM respectively.

3\% noise was applied to T1-weighted MR phantom Fig. 15. (a).

Fig. 15(b), (c) and (d) shows a comparison of segmentation results between FCM, SFCM, and IFCM. From these images, we can see that traditional FCM was unable to correctly classify the images. IFCM produced slightly better results than SFCM due to its ability to cope with noise. Fig. 16(a) shows T1 weighted brain MRI. Fig. 16(b), (c) and (d) shows a segmentation results among FCM, SFCM, and IFCM.

Fig. 17(a) shows noisy T2 weighted brain MR image. The Segmentation results after applying FCM, SFCM, and IFCM are shown in Fig. 17(b), (c) and (d) respectively.

These indicate that whereas FCM is unable to segment the slice correctly, and noise still remains present, IFCM produced the better result with slight left over noise. The results presented in this paper are groundwork and further clinical evaluation is required. 


\section{CONCLUSION}

This paper aims at to give an overview of the MR brain image segmentation problem and discuss various techniques. In this paper, segmentation algorithms FCM, KFCM, SFCM, and IFCM have been reviewed. Traditional FCM, as not being vigorous for noisy images, appears to be a popular segmentation method for medical images. Many extended algorithms based on FCM have been developed to solve this problem. The IFCM algorithm produces better result as compared to FCM, KFCM, and SFCM. The result founded in this paper is preliminary and further clinical evaluation is required. Extensive experiments using MR images generated by the Brain Web simulator [19] data have been used to evaluate the proposed methods. The future experiments may be useful in the direction of changing distance in FCM for better results in segmentation and focusing on Fuzzy min max neural model.

\section{ACKNOWLEDGMENTS}

First, I thank to Dr. M. H. Kondekar for his continuous support and valuable guidance. I thank to D.H. MAHAMUNI and J.R KAWALE for the paper that encouraged, as well as for the lots of discussions carried on long-distance via communication.

\section{REFERENCES}

[1] D. Tian, L. Fan (2007), "A brain MR images segmentation method based on SOM neural network," In: The $1^{\text {st }}$ Int. Conf. bioinformatics and biomedical engineering, pp 686-689.

[2] X. Han, B. Fischl (2007), "Atlas renormalization for improved brain MR image segmentation across scanner platforms," IEEE Trans. Med. Imag., 26(4):479-486.

[3] D. L. Pham, C. Y. Xu, J. L. Prince, "A survey of current methods in medical image segmentation," Ann Rev. Biomed. Eng., 2 (2000), pp.315-37 [Technical report version, JHU/ECE 99-01, Johns Hopkins University].

[4] M. N. Ahmed, S. M. Yamany, N. Mohamed, A. A. Farag, and T. Moriarty (2002), "A modified fuzzy Cmeans algorithm for bias field estimation and segmentation of MRI data," IEEE Trans. on Med. Imag., 21(3), pp.193-199.

[5] L. Ma and R. C. Staunton (2007), "A modified fuzzy cmeans image segmentation algorithm for use with uneven illumination Patterns," Pattern Recognition, 40(11), pp.3005-3011.

[6] S. Shen, W. A. Sandham, M. H. Granat, and A. Sterr (2005), "MRI fuzzy segmentation of brain tissue using neighborhood attraction with neural-network optimization". IEEE Trans. On Information Technology in Biomedicine, vol. 9, no. 3, September 2005459.

[7] K. S. Chuang, H. L. Hzeng, S. Chen, J. Wu, T. J. Chen, (2006), "Fuzzy c-means clustering with spatial information for image segmentation," Comp. Med. Imag. and Graphics, 30, pp.9-15.

[8] C. L. Li, D. B. Goldgof, L. O. Hal, "Knowledge-based Classification and tissue labeling of MR images of human brain," IEEE Trans. Med. Imag., vol. 12, no. 4, pp. 740-750, Apr. 1993.
[9] L. O. Hall, A. M. Bensaid, L. P. Clarke, R. P. Velthuizen, M. S. Silbiger, J. C. Bezdek , "A comparison of neural network and fuzzy clustering techniques in segmenting magnetic resonance images of the brain," IEEE Trans. Neural Netw., vol. 3, no. 5, pp. 672-682, Sep. 1992.

[10] B. Alfano, A. Brunetti, et al. "Unsupervised, automated segmentation of the normal brain using multispectral relaxometric magnetic resonance approach," Magn. Reson. Med., Vol. 37, 1997, pp. 84-93.

[11] N. Guillermo, Abras and Virginia L. Ballarin, "A Weighted K-means Algorithm applied to Brain Tissue Classification".

[12] R. De La Paz, E. Herskovits, V. Gesu, et al. "Cluster analysis of medical magnetic resonance images MRI data: Diagnostic application and evaluation," SPIE Extracting Meaning from Complex Data: Processing, Display, Interaction, Vol. 1259, 1990, pp. 176-181.

[13] Y. Wei, J. Fritts, and S. Fangting, "A hierarchical image segmentation algorithm," ICME '02. Proc. IEEE Int. Conf., Vol. 2, 2002, pp. 221-224.

[14] J. C. DUNN, "A fuzzy relative of the ISODATA process and its use in detecting compact well-separated clusters," J. Cybern., vol. 3, pp. 32-57, 1973.

[15] J. C. BEZDEK, Pattern Recognition with Fuzzy Objective Function Algorithms, Plenum Press, New York 1981.

[16] D. Q. Zhang and S. C. Chen, "A novel kernelized fuzzy c-means algorithm with application in medical image segmentation," Artificial Intelligence in Medicine, vol. 32, pp. 37-50, 2004.

[17] D. Q. Zhang, "Kernel-based fuzzy clustering incorporating spatial constraints for image segmentation," in Proc. of the $2^{\text {nd }}$ Int. Conf. on Mach. Lear. And Cyb., pp. 2189-2192 Vol. 4, 2003.

[18] R. Pohle and K. D. Toennies, "Segmentation of medical images using adaptive region growing," Proc. SPIEMed. Imag., vol. 4322, pp. 1337-1346, 2001.

[19] Brain Web [Online]. Available: ww.bic.mni.mcgill.ca/brainweb/

[20] Yong Yang, "Image Segmentation by Fuzzy C-Means Clustering Algorithm with a Novel Penalty Term," Computing and Informatics, Vol. 26, 2007, 17-31

[21] Keh-Shi Chuang, Hong-Long Tzeng, Sharon Chen, Jay $\mathrm{Wu}$, and Tzong-Jer Chen, "Fuzzy c-means clustering with spatial information for image segmentation," Comput. Med. Imag. and Graphics 30 (2006) 9-15.

[22] Aimin Yang, Lingmin Jiang, Yongmei Zhou, "A KFCM-based Fuzzy Classifier".

[23] Xiao-li Jin, Tu-sheng Lin Liang Liao and Teng Wang, "Multi-Spectral MRI Brain Image Segmentation Based on Kernel Clustering Analysis," 2012 Int. Conf. on System Eng. and Modeling (ICSEM 2012) IPCSIT vol. 34 (2012). 
International Journal of Computer Applications (0975 - 8887)

Volume 157 - No 8, January 2017

[24] Maryam Rastgarpour and Jamshid Shanbehzadeh, "A New Kernel-Based Fuzzy Level Set Method for Automated Segmentation of Medical Images in the Presence of Intensity In homogeneity".

[25] D. L. Pham, J. L. Prince, “An adaptive fuzzy C-means algorithm for image segmentation in the presence of intensity inhomogeneities," Pattern Recognit Lett 1999; 20(1):57-68.

[26] Y. A. Tolias, S. M. Panas, "On applying spatial constraints in fuzzy image clustering using a fuzzy rule- based system," IEEE Signal Proc. Lett., 1998; 5(10):245-7.

[27] Y. A. Tolias, S. M. Panas, "Image segmentation by a fuzzy clustering algorithm using adaptive spatially constrained membership functions," IEEE Trans. Syst, Man., Cybernet Part A 1998; 28(3):359—69.

[28] AWC. Liew, S. H. Leung, W. H. Lau, "Fuzzy image clustering incorporating spatial continuity," IEE Proc. Vis. Image Signal Proc. 2000; 147(2):185—92. 